\title{
Impact Sound Insulation and Viscoelastic Properties of Resilient Materials made from Recycled Tyre Granules
}

\author{
Francesco Asdrubali and Francesco D’Alessandro \\ University of Perugia, Department of Industrial Engineering, Via Duranti, 67, Perugia, Italy
}

(Received 13 September 2010, accepted 24 January 2011)

The disposal of used tyres is a significant environmental problem in developed countries. The production of acoustic materials from rubber crumbs can therefore represent a valid alternative to incineration or to the disposal of used tyres into landfill.

The goal of the paper is to analyze and optimize the manufacturing process of impact sound insulating materials made of recycled tyre granules mixed with binders. Several prototypes were manufactured in laboratory by means of the developed process and tested in order to evaluate their dynamic stiffness. The influence of grain size, binder concentration and density of the sample was investigated. Thanks to the measured values of the viscoelastic properties, it was possible to model the new materials and to estimate the indexes of impact sound reduction $\Delta \mathrm{L}_{\mathrm{W}}$.

Finally, the prototypes showing the best properties were produced in bigger size and tested in two overlapping reverberating rooms according to the standard ISO 140-8. Results confirm that the materials manufactured through the developed process have satisfactory acoustic properties, comparable to the ones of commercially available materials.

\section{INTRODUCTION}

Energy consumption in the building sector can reach up to $40 \%$ of the total energy demand of an industrialized country. For this reason, green building strategies can be extremely effective as far as fossil fuel savings and greenhouse gas reduction. Sustainable materials made from natural or recycled materials can play an important role, since less energy is generally required for their production than that needed for producing conventional materials.

In the last years a great attention has been focused on green materials, especially in the building sector. Many research centres have developed new sustainable materials, in many cases with interesting thermal and acoustical properties. ${ }^{1}$ In addition, the public sector has started to consider these materials. In Italy, for instance, many municipalities have introduced specific recommendations into building regulations to increase the use of ecological materials in new constructions, allowing a reduction of construction taxes.

In particular, natural fibres are increasingly considered as alternatives to synthetic ones, in order to combine high acoustic and thermal performance with a low impact on the environment and human health. Recycled materials, such as recycled plastic fibres and recycled rubber mats, can even be regarded as a sustainable alternative, as they contribute to lower waste production and use of raw materials.

Within this context, recycled tyre granules have been recently proposed for manufacturing acoustic insulating and absorbing materials, to be used for noise control in buildings and road barriers. Materials made of rubber crumbs usually have high porosity, and they consequently show good sound absorbing properties over a wide frequency range. Furthermore, the use of these materials in insulating underlays shows satisfactory performance for acoustic impact insulation.

The development of novel acoustic materials made from end-of-life tyres can possibly be a solution for the disposal of these materials. Used tyres represent in fact a huge amount of material to be disposed. Estimates are that more than 250 million post-consumer tyres are accumulated each year in the fifteen Member States of the European Union, and comparable amounts are amassed in Eastern Europe, North America, Latin America, Asia, and the Middle-East, totalling more than 1,000 million additional tyres per year. ${ }^{2}$ The largest reuse market is tyre-derived fuel, followed by civil engineering applications such as highway embankments; however, the production of rubber crumbs is growing, and the subsequent applications are gaining importance. ${ }^{3}$

The goal of this paper is to develop an innovative process using recycled tyre granules to produce materials for acoustic applications, in particular resilient insulating layers. Since the grains themselves show no mechanical strength, it is necessary to mix them with an adequate binder and to consolidate the compound in order to create a solid structure. The parameters that mainly influence the properties of this kind of material are grain size, binder type and concentration, compaction ratio, and final thickness, so an optimized manufacturing process has to be developed. ${ }^{4}$

After a brief literature review, this paper focuses on the damping properties of various sample materials made from tyre granules and presents the chemical characterization of the materials, the description of the manufacturing process, and the results of the experimental campaign and modelling process carried out at the Acoustic Laboratory of the University of Perugia. 